\title{
SKEW PRODUCTS OF DYNAMICAL SYSTEMS
}

\author{
BY \\ EIJUN KIN
}

\begin{abstract}
In 1950-1951, H. Anzai introduced a method of skew products of dynamical systems in connection with isomorphism problems in ergodic theory. There is a problem to give a necessary and sufficient condition under which an ergodic skew product dynamical system has pure point spectrum. For the special case, translations on the torus, he gave a partial answer for this question. However, this problem has been open in the general case.

In the present paper, we generalize the notion of skew products proposed by Anzai and give a complete answer for this problem.
\end{abstract}

1. Introduction. A method of skew products of dynamical systems, which is powerful to examine the geometrical structures of trajectories in dynamical systems, was first studied by $\mathbf{H}$. Anzai [1] in connection with isomorphy problems in ergodic theory. In order to explain the method of skew products, we now take the typical one. Let $\left\{T_{t}:-\infty<t<\infty\right\}$ be a measurable flow on a probability measure space $\left(X, \mathscr{F}_{X}, m_{X}\right),\left\{S_{t}:-\infty<t<\infty\right\}$ be a measurable flow on another probability measure space $\left(Y, \mathscr{F}_{Y}, m_{Y}\right)$ and $\left(\Omega^{*}, \mathscr{B}^{*}, m^{*}\right)$ be the direct product measure space of $\left(X, \mathscr{F}_{X}, m_{X}\right)$ and $\left(Y, \mathscr{F}_{Y}, m_{Y}\right)$. Further, let $a(x)$ be a positive integrable function on $X$. Put

$$
\alpha(t, x)=\int_{0}^{t} a\left(T_{s} x\right) d s,
$$

then the function $\alpha(t, x)$ is measurable in $(t, x)$ and possesses the additivity $\alpha(t+s, x)=\alpha(t, x)+\alpha\left(s, T_{t} x\right)$ for any $t, s$ and for almost all $x \in X$ (cf. Hopf [2, p. 43]). We assume that the set $\left\{(t, x, y): S_{\alpha(t, x)} y \in E\right\}$ belongs to the direct product $\sigma$-field $\mathscr{B}^{1} \otimes \mathscr{F}_{X} \otimes \mathscr{F}_{Y}$ for every $E \in \mathscr{F}_{Y}$, where $\mathscr{B}^{1}$ is the Borel $\sigma$-field on the real line $R^{1}$. Let us set

$$
Z_{t}(x, y)=\left(T_{t} x, S_{\alpha(t, x)} y\right),
$$

then we obtain a measurable flow $\left\{Z_{t}:-\infty<t<\infty\right\}$ on $\left(\Omega^{*}, \mathscr{B}^{*}, m^{*}\right)$, and such a flow $\left\{Z_{t}\right\}$ is called an ordinary simple skew product flow of $\left\{T_{t}\right\}$ and $\left\{S_{t}\right\}$ with onedimensional parameter.

The purpose of the present paper is to generalize this fact and to study the ergodic and the spectral properties of such dynamical systems.

Received by the editors July 8, 1970.

AMS 1969 subject classifications. Primary 2870.

Key words and phrases. Generalized flow, simple skew product, $N$-fold skew product, proper value function, Borel cycle, homology, quasigroup, generalized canonical flow, exact sequence, stability.

Copyright (c) 1972, American Mathematical Society 
Skew product dynamical systems have not only a noticeable interesting property which cannot be observed in the direct product dynamical systems (see Anzai [3] and Kin [4]) but a treasured application to the random ergodic theory (cf. Anzai [3], Kakutani [5], Ryll-Nardzewski [6], Gładysz [7], [8] and Kin [9]). But we do not discuss them minutely in our present paper. In $\$ 2$ and $\S 3$ we are going to generalize the Anzai definition of a skew product dynamical system and to prepare the basic concepts for the later use. In $\S 4$ the criteria for ergodicity and proper values are considered for a generalized simple skew product flow. In $\$ 5$ we discuss the spectral properties of such a flow. In $\S 6$ we extend the considerations of $\S 3, \S 4$ and $\$ 5$ to the case of the generalized $N$-fold skew product flows.

It is easy to see that two ordinary simple skew product flows $\left\{Z_{t}^{(1)}: t \in R^{d}\right\}$ and $\left\{Z_{t}^{(2)}: t \in R^{d}\right\}$ with $d$-dimensional parameter built up respectively by the functions $\alpha_{1}(t, x)$ and $\alpha_{2}(t, x)$ such that $\alpha_{1}(t, x)$ is homologous to $\alpha_{2}(t, x)$ are spatially isomorphic to each other. Conversely, one can ask whether $\alpha_{1}(t, x)$ is homologous to $\alpha_{2}(t, x)$ or not when the skew product flow $\left\{Z_{t}^{(1)}\right\}$ built up by $\alpha_{1}(t, x)$ is isomorphic to the skew product flow $\left\{Z_{t}^{(2)}\right\}$ built up by $\alpha_{2}(t, x)$. We have not been able to show the complete answer to this question, but a partial positive answer together with the brief informations about the property and the application stated above is established in the final section.

The author would like to express his deep thanks to Professor $\mathrm{H}$. Nomoto and Dr. I. Kubo for fruitful discussions and suggestions.

2. Preliminaries. By a probability measure space we mean a triple $\left(X, \mathscr{F}_{X}, m_{X}\right)$ where $X$ is a nonempty abstract set, $\mathscr{F}_{X}$ is the Borel $\sigma$-field of subsets of $X$ and $m_{X}$ is a probability measure on $\mathscr{F}_{X}$. Let $G$ be a locally compact separable Abelian group and $\mathscr{B}$ the topological Borel $\sigma$-field on $G$.

Definition 2.1. A system $\left\{T_{g}: g \in G\right\}$ of transformations on $\left(X, \mathscr{F}_{X}, m_{X}\right)$ is called a generalized measurable flow on the space if the following conditions are fulfilled:

(i) for each $g \in G, T_{g}$ is an automorphism of the space,

(ii) $T_{g_{1}} \cdot T_{g_{2}}=T_{g_{1} \cdot g_{2}}$ and $T_{e}=$ identity ( $e$ : the unit element of $G$ ),

(iii) the mapping $(g, x) \rightarrow T_{g} x$ from $G \otimes X$ to $X$ is $\mathscr{B} \otimes \mathscr{F}_{X}$-measurable $\left({ }^{1}\right)$.

Take $G=R^{d}$. Then $\left\{T_{t}: t \in R^{d}\right\}$ is said to be an ordinary measurable flow with $d$-dimensional parameter. When $G=Z^{d}$, where $Z^{1}$ denotes the set of all integers, $\left\{T_{n}: n \in Z^{d}\right\}$ is called an ordinary $d$-parameter discrete flow. We say that a generalized flow $\left\{T_{g}\right\}$ is ergodic provided that if $f$ is measurable and if $f\left(T_{g} x\right)=f(x)$ for almost all $x \in X$ and all $g \in G$, then $f$ is constant almost everywhere (i.e., the only invariant functions are constant a.e.). A generalized flow $\left\{T_{g}\right\}$ is said to have a proper value function $\lambda$ if there exists an integrable function $f$ such that $f\left(T_{g} x\right)$

(1) Where no confusion can arise, we shall employ the symbol $\left\{T_{g}\right\}$ instead of $\left\{T_{g}: g \in G\right\}$ throughout this paper. 
$=\lambda(g) \cdot f(x)$ for almost all $x \in X$ and all $g \in G$, where $\lambda$ is a continuous homomorphism from $G$ into the unit circle in the complex plane. Then we call the function $f$ a proper function of $\left\{T_{g}\right\}$ corresponding to $\lambda$. Evidently $\hat{G} \supseteq s\left(\left\{T_{g}\right\}\right)$, where $\hat{G}$ stands for the character group of $G$ and $s\left(\left\{T_{g}\right\}\right)$ stands for the set of all proper value functions of $\left\{T_{g}\right\}$.

DefinItion 2.2. We say that a generalized flow $\left\{T_{g}\right\}$ on $\left(X, \mathscr{F}_{X}, m_{X}\right)$ has pure point spectrum if the class of proper functions of $\left\{T_{g}\right\}$ becomes a complete orthogonal basis of $L_{2}(X)$ which is the Hilbert space consisting of complex valued square integrable functions defined on $X$.

The argument for a measurable flow on an abstract probability measure space can be changed to that of a measurable flow on a compact Abelian group equipped with a suitable regular Borel measure. By a slight modification of the method of Foias [10], we can easily show the following

THEOREM 2.1. For a generalized measurable flow $\left\{T_{g}: g \in G\right\}$ on a probability measure space $\left(\Omega, \mathscr{F}_{\Omega}, m_{\Omega}\right)$, there exist a compact Abelian group $X$ endowed with a normalized regular Borel measure $m_{X}$ and a generalized measurable flow $\left\{S_{g}: g \in G\right\}$ on $X$ which is homomorphic to $\left\{T_{g}\right\}$.

Proof. Cf. Kin [11].

The well-known theorem of von Neumann [12] assures that if $\left\{T_{g}\right\}$ is ergodic and has pure point spectrum, then the measure $m_{X}$ in the theorem can be taken to be a normalized Haar measure. Now let us mention a few examples concerning generalized flows.

EXAMPLE 2.1. Suppose $E$ is an infinite-dimensional real nuclear space, $M$ is its completion by the continuous Hilbertian norm of $E, X$ is the conjugate space of $E$ and $G=O(M)$, where $O(M)$ is the group formed by all linear and orthogonal operators acting on $M$. Then we obtain a generalized flow $\left\{T_{g}: g \in G\right\}$ on the probability measure space $\left(X, \mathscr{F}_{X}, m_{X}\right)$ in which $m_{X}$ is a Gaussian measure defined on the $\sigma$-field $\mathscr{F}_{X}$ generated by all cylinder sets of $X$. For the existence of such a flow, see Nomoto [13].

EXAMPLE 2.2 . Let $(\Omega, \mathscr{B}, m)$ be a probability measure space and $I$ an infinite set, for instance, the set of all integers or all real numbers. We consider a group $G$ of all permutations of $I$ and for each $g \in G$, we define a $G$-index automorphism $T_{g}$ of the product space $\left(\bigotimes_{i \in I} \Omega, \bigotimes_{i \in I} \mathscr{B}, \bigotimes_{i \in I} m\right)$ as follows:

$$
T_{g}\left(\omega_{i}: i \in I\right)=\left(\omega_{g(i)}: i \in I\right), \quad\left(\omega_{i}: i \in I\right) \in \bigotimes_{i \in I} \Omega .
$$

Then $\left\{T_{g}: g \in G\right\}$ is a generalized flow on the product space. For ergodicity of such a flow, we refer the reader to Kin [14].

3. Generalized simple skew product flows. The aim of this section is to generalize the Anzai's formulation about skew products to multi-parameter cases. 
To begin with, we shall define some fundamental concepts provided for our later employment. There is given a generalized measurable flow $\left\{T_{g}\right\}$ on a $\sigma$-finite measure space $\left(X, \mathscr{F}_{X}, m_{X}\right)\left(m_{X}\right.$ is a $\sigma$-finite measure on $\left.\mathscr{F}_{X}\right)$. Suppose a function $\alpha(g, x)$ is defined on $G \otimes X$ and takes its values in $G$.

Definition 3.1. A function $\alpha(g, x)$ is said to be a Borel cycle with respect to the flow $\left\{T_{g}\right\}$ provided that the function $\alpha(g, x)$ is Borel measurable in $(g, x)$ and satisfies the relation:

$$
\alpha\left(g_{1} \cdot g_{2}, x\right)=\alpha\left(g_{1}, x\right) \cdot \alpha\left(g_{2}, T_{g_{1}} x\right)
$$

for any $g_{1}, g_{2} \in G$ and for almost all $x \in X$.

We consider the collection $\Gamma(X)$ of measurable function $\theta(x)$ defined on $X$ taking its values in the unit circle in the complex plane, and by $H(X)$ we mean the family of complex valued measurable functions $\varphi(g, x)$ defined on $G \otimes X$ such that, for some $\theta(x) \in \Gamma(X), \varphi(g, x)=\theta\left(T_{g} x\right) \cdot \theta(x)^{-1}$ holds almost everywhere on $X$ for all $g \in G$.

Definition 3.2. Let $\psi_{1}(g, x)$ and $\psi_{2}(g, x)$ be two complex valued $(g, x)$-measurable functions with the absolute value one. With respect to the flow $\left\{T_{g}\right\}$, the function $\psi_{1}(g, x)$ is said to be homologous to the function $\psi_{2}(g, x)$ if $\psi_{1}(g, x)$ - $\psi_{2}(g, x)^{-1}$ belongs to $H(X)$.

Suppose there is given a family $\left\{S_{(g, x)}: g \in G\right\}$ of transformations on another $\sigma$-finite measure space $\left(Y, \mathscr{F}_{Y}, m_{Y}\right)$ satisfying that

(i) for each $(g, x) \in G \otimes X, S_{(g, x)}$ is an automorphism of $\left(Y, \mathscr{F}_{Y}, m_{Y}\right)$,

(ii) $S_{\left(g_{1} \cdot g_{2}, x\right)} y=S_{\left(g_{2}, T_{g_{1}} x\right)} \cdot S_{\left(g_{1}, x\right)} y$ holds almost everywhere on $X \otimes Y$ for any $g_{1}, g_{2} \in G$,

(iii) both the set $\left\{(g, x, y): S_{(g, x)} y \in E\right\}$ and the set $\left\{(g, x, y): S_{(g, x)}^{-1} y \in E\right\}$ belong to $\mathscr{B} \otimes \mathscr{F}_{X} \otimes \mathscr{F}_{Y}$, where $E$ is an arbitrary $\mathscr{F}_{Y}$-measurable set $\left({ }^{2}\right)$.

Denote by $\left(\Omega^{*}, \mathscr{B}^{*}, m^{*}\right)$ the direct product measure space of the two spaces $\left(X, \mathscr{F}_{X}, m_{X}\right)$ and $\left(Y, \mathscr{F}_{Y}, m_{Y}\right)$. Using $\left\{T_{g}\right\}$ and $\left\{S_{(g, x)}\right\}$ mentioned above, we define the transformation group $\left\{Z_{g}: g \in G\right\}$ as follows:

$$
Z_{g}(x, y)=\left(T_{g} x, S_{(g, x)} y\right)
$$

for all $g \in G$ and all $(x, y) \in \Omega^{*}$. Then it follows from the hypotheses granted to $\left\{T_{g}\right\}$ and $\left\{S_{(g, x)}\right\}$ that $\left\{Z_{g}\right\}$ is a measurable flow. We call this $\left\{Z_{g}\right\}$ a generalized 1-fold skew product (or a generalized simple skew product) flow on the $\sigma$-finite measure space $\left(\Omega^{*}, \mathscr{B}^{*}, m^{*}\right)$ built up by $\left\{T_{g}\right\}$ and $\left\{S_{(g, x)}\right\}$. If we take $G=R^{d}\left(G=Z^{d}\right)$, $\left\{Z_{t}: t \in R^{d}\right\}\left(\left\{Z_{n}: n \in Z^{d}\right\}\right)$ is called an ordinary simple skew product (discrete) flow with $d$-dimensional parameter.

EXAMPLE 3.1. Let $\left\{T_{t}: t \in R^{d}\right\}$ and $\left\{S_{t}: t \in R^{d}\right\}$ be ordinary measurable flows on $\left(X, \mathscr{F}_{X}, m_{X}\right)$ and $\left(Y, \mathscr{F}_{Y}, m_{Y}\right)$ respectively. Furthermore, let $\alpha(t, x)$ be a Borel

$\left.{ }^{2}\right)$ We say that if the family $\left\{S_{(g, x)}\right\}$ satisfies the condition (ii), this family is a quasigroup with respect to $\left\{T_{g}\right\}$. 
cycle with respect to $\left\{T_{t}\right\}$. Under the consideration of the measurability condition (iii), the ordinary simple skew product flow $\left\{Z_{t}\right\}$ on $\Omega^{*}$ is given by

$$
Z_{t}(x, y)=\left(T_{t} x, S_{\alpha(t, x)} y\right) .
$$

When $d=1, X=Y=R^{1}$ and when both $\left\{T_{t}\right\}$ and $\left\{S_{t}\right\}$ are the 1-translations on $R^{1}$, the flow $\left\{Z_{t}\right\}$ runs as follows:

$$
Z_{t}(x, y)=(x+t, y+\alpha(t, x)) .
$$

In this case, it was shown by Anzai [3] that for any Borel cycle $\alpha(t, x)$ with respect to $\left\{T_{t}\right\}$, there exists a measurable function $\beta(x)$ on $X$ such that $\alpha(t, x)=\beta\left(T_{t} x\right)-\beta(x)$. Moreover, he proved that, if $X=[0,1)$, for any Borel cycle $\alpha(t, x)$ with respect to a periodic motion $\left\{T_{t}\right\}$ on $X$ with frequency one, there exist a constant $c$ and a measurable function $\beta(x)$ on $X$ such that

$$
\alpha(t, x)-c \cdot t=\beta\left(T_{t} x\right)-\beta(x)(\bmod 1) .
$$

EXAMPLE 3.2. Let $T$ be an automorphism of $\left(X, \mathscr{F}_{X}, m_{X}\right)$ and $\left\{S_{g}: g \in G\right\}$ a generalized measurable flow on $\left(Y, \mathscr{F}_{Y}, m_{Y}\right)$. We assume that $\alpha(x)$ is a measurable function defined on $X$ taking its values in $G$. Then we have a skew product automorphism $Z$ of $\left(\Omega^{*}, \mathscr{B}^{*}, m^{*}\right)$ defined as follows:

$$
Z(x, y)=\left(T x, S_{\alpha(x)} y\right) .
$$

Of course, we assume that $\left\{S_{\alpha(x)}\right\}$ satisfies the measurability condition similar to (iii) (cf. Anzai [3]).

In the case of $G=R^{1}$ and $X=Y=R^{1}$, if $T$ is the $\delta$-translation and if $\left\{S_{t}\right\}$ is the 1-translation on $R^{1}$, then the automorphism $Z$ runs as follows:

$$
Z(x, y)=(x+\delta, y+\alpha(x)) \text {. }
$$

Anzai [1] studied the ergodicity, the mixing and the spectral properties of such a skew product automorphism $Z$ in the case of $X=Y=[0,1)$.

EXAMPLE 3.3. Let $\left\{T_{g}: g \in G\right\}$ be a generalized measurable flow on $\left(X, \mathscr{F}_{X}, m_{X}\right)$ and $Y$ a locally compact Abelian group with Haar measure. Suppose $\alpha(g, x)$ is a Borel cycle with respect to $\left\{T_{g}\right\}$ and $\pi$ is a homomorphism from $G$ into $Y$. On making use of $\pi$, we have a generalized canonical flow $\left\{S_{g}: g \in G\right\}$ on $Y$ given by $S_{g} y=\pi(g) \cdot y$. We assume further that $\left\{S_{\alpha(g, x)}\right\}$ satisfies the condition (iii). Then we obtain the generalized simple skew product flow $\left\{Z_{g}: g \in G\right\}$ defined as follows:

$$
Z_{g}(x, y)=\left(T_{g} x, \Delta(g, x) \cdot y\right),
$$

where $\Delta(g, x)=\pi(\alpha(g, x))$, i.e. $\Delta=\pi \circ \alpha$.

The theorem of von Neumann implies that in order to study the generalized simple skew product flow $\left\{Z_{g}\right\}$ of a measurable flow $\left\{T_{g}\right\}$ on a probability measure space and an ergodic measurable flow $\left\{S_{g}\right\}$ on another probability measure space with pure point spectrum, it is sufficient to analyze the skew product flow $\left\{Z_{g}\right\}$ given in Example 3.3. 
4. Point spectra. In this section we deal with the generalized simple skew product flows as given in Example 3.3, and we shall characterize the ergodicity and the proper value functions of such flows by means of the homology of Borel cycles.

Let $\left(X, \mathscr{F}_{X}, m_{X}\right)$ be a probability measure space on which a generalized measurable flow $\left\{T_{g}\right\}$ is given, and let $Y$ be a compact Abelian group equipped with the normalized Haar measure $m_{Y}$, on which a generalized canonical flow $\left\{S_{g}\right\}$ is given. Suppose $\alpha(g, x)$ is a Borel cycle with respect to $\left\{T_{g}\right\}$ and $\left\{S_{\alpha(g, x)}\right\}$ satisfies the measurability condition (iii) in $\S 3$. We denote by $\hat{Y}$ the character group of $Y$ and note that the function $\Delta(g, x)$ given in Example 3.3 is a Borel cycle.

THEOREM 4.1. A generalized simple skew product flow $\left\{Z_{g}\right\}$ of $\left\{T_{g}\right\}$ and $\left\{S_{g}\right\}$ built up by $\Delta(g, x)$ is ergodic if and only if $\left\{T_{g}\right\}$ is ergodic and for any $\hat{y} \in \hat{Y}$ with $\hat{y} \neq \hat{e}$ ( $\hat{e}$ : the unit character), $\hat{y}(\Delta(g, x)) \notin H(X)$, that is, the Borel cycle $\hat{y} \circ \Delta$ is not homologous to one.

Proof. To prove the "if" part, we suppose $\left\{T_{g}\right\}$ is ergodic and $\hat{y}(\Delta(g, x)) \notin H(X)$ for any $\hat{y} \in \hat{Y}$ with $\hat{y} \neq \hat{e}$. If the flow $\left\{Z_{g}\right\}$ is not ergodic, then there exists a nonconstant function $h(x, y)$ in $L_{2}\left(\Omega^{*}\right)$ such that

$$
h\left(Z_{g}(x, y)\right)=h(x, y)
$$

holds for all $g \in G$ and almost all $(x, y) \in \Omega^{*}$. Consider the function $f(\hat{y} ; x)$ defined by

$$
f(\hat{y} ; x)=\int_{Y} h(x, y) \hat{y}(y) d m_{Y}(y)
$$

for any $\hat{y} \in \hat{Y}$. From (4.1) and (4.2), we get

$$
f(\hat{y} ; x)=\hat{y}(\Delta(g, x))^{-1} f\left(\hat{y} ; T_{g} x\right) .
$$

On noticing that $\hat{Y}$ is a complete orthonormal system of $L_{2}(Y)$, the ergodicity of $\left\{T_{g}\right\}$ implies that there exists a character $\hat{y}_{0}$ in $\hat{Y}$ with $\hat{y}_{0} \neq \hat{e}$ such that

$$
\left|f\left(\hat{y}_{0} ; x\right)\right|=\left|f\left(\hat{y}_{0} ; T_{g} x\right)\right|=c\left(\hat{y}_{0}\right)
$$

where $c\left(\hat{y}_{0}\right)$ is some positive constant. Fix such a character $\hat{y}_{0}$. There exists a function $\theta(x)$ in $\Gamma(X)$ such that

$$
f\left(\hat{y}_{0} ; x\right)=c\left(\hat{y}_{0}\right) \cdot \theta(x) .
$$

By replacing the function $f(\hat{y} ; x)$ in (4.3) by the function $f\left(\hat{y}_{0} ; x\right)$ in (4.5), we obtain

$$
\hat{y}_{0}(\Delta(g, x))=\theta\left(T_{g} x\right) \cdot \theta(x)^{-1},
$$

which contradicts our assumption.

Conversely, suppose $\left\{Z_{g}\right\}$ is ergodic. It is clear that $\left\{T_{g}\right\}$ becomes ergodic. If $\hat{y}(\Delta(g, x)) \in H(X)$ for some $\hat{y} \in \hat{Y}$ with $\hat{y} \neq \hat{e}$, there exists a function $\theta(x)$ in $\Gamma(X)$ preserving the equality (4.6). For these $\theta(x)$ and $\hat{y}(y)$, take $h(x, y)=\theta(x) \hat{y}(y)^{-1}$. 
Although $h(x, y)$ in $L_{2}\left(\Omega^{*}\right)$ is not a constant function, it is invariant under the flow $\left\{Z_{g}\right\}$. Consequently, these contradictions complete the proof of the theorem.

REMARK 4.1. If for an ordinary measurable flow $\left\{T_{t}: t \in R^{d}\right\}$ on $\left(X, \mathscr{F}_{X}, m_{X}\right)$ with $d$-dimensional parameter, there exist a $d$-dimensional real vector $\lambda$ in $R^{d}$ and a function $f(x)$ in $L_{2}(X)$ such that $f\left(T_{t} x\right)=\exp i\langle\lambda, t\rangle \cdot f(x)$ for almost all $x \in X$ and all $t \in R^{d}, \lambda$ and $f$ are called a proper value and a proper function corresponding to $\lambda$ respectively. By putting $\lambda(t)=\exp i\langle\lambda, t\rangle$, where $\langle\lambda, t\rangle$ denotes the inner product of $\lambda$ and $t$ in the space $R^{d}$, the proper value $\lambda$ may be understood as a proper value function of $\left\{T_{t}\right\}$ (see $\S 2$ ). We recall that the flow $\left\{T_{t}\right\}$ is said to have pure point spectrum if $L_{2}(X)$ can be spanned by the orthogonal system consisting of its proper functions.

COROllaRY 4.1. Let $\left\{T_{t}: t \in R^{d}\right\}$ be an ordinary measurable flow on $\left(X, \mathscr{F}_{X}, m_{X}\right)$ and $\left\{S_{t}: t \in R^{d}\right\}$ an ordinary measurable flow on another probability measure space $\left(Y, \mathscr{F}_{Y}, m_{Y}\right)$ with pure point spectrum. Moreover, let $\alpha(t, x)$ be a Borel cycle with respect to $\left\{T_{t}\right\}$. In consideration of the measurability condition (iii) in $\S 3$, the ordinary simple skew product flow $\left\{Z_{t}\right\}$ of $\left\{T_{t}\right\}$ and $\left\{S_{t}\right\}$ built up by $\alpha(t, x)$ is ergodic if and only if $\left\{T_{t}\right\}$ is ergodic and, for any nonzero proper value $r$ of $\left\{S_{t}\right\}$, exp $i\langle r, \alpha(t, x)\rangle \notin H(X)$.

COROllary 4.2. When we take $Y=[0,1)$ and the flow $\left\{S_{t}\right\}$ to be a periodic motion on $Y$ with frequency $a$ in $R^{d}$, i.e. $S_{t} y=y+\langle a, t\rangle(\bmod 1)$ in the preceding corollary, the ordinary simple skew product flow $\left\{Z_{t}\right\}$ is ergodic if and only if $\left\{T_{t}\right\}$ is ergodic and for any nonzero integer $p, \exp i p \cdot\langle a, \alpha(t, x)\rangle \notin H(X)$.

The next corollary generalizes the criteria due to Anzai [1].

COROllaRY 4.3. Let $T$ be an automorphism of $\left(X, \mathscr{F}_{X}, m_{X}\right)$ and $\left\{S_{t}: t \in R^{d}\right\}$ an ordinary d-parameter measurable flow on $\left(Y, \mathscr{F}_{Y}, m_{Y}\right)$ with pure point spectrum. Further, let $\alpha(x)$ be a measurable function defined on $X$ taking values in $R^{d}$. The skew product automorphism $Z$ of $T$ and $\left\{S_{t}\right\}$ built up by $\alpha(x)$ is ergodic if and only if $T$ is ergodic and $\exp i\langle r, \alpha(x)\rangle \notin H(X)$ holds for any nonzero proper value $r$ of $\left\{S_{t}\right\}$.

THEOREM 4.2. Let $\left\{Z_{g}\right\}$ be the generalized simple skew product flow as in Theorem 4.1, and suppose $\left\{T_{g}\right\}$ is ergodic. $\lambda$ is a proper value function of $\left\{Z_{g}\right\}$ if and only if there exists a character $\hat{y}$ in $\hat{Y}$ such that $\hat{y}(\Delta(g, x))$ is homologous to $\lambda(g)$.

Proof. Suppose $\lambda \in s\left(\left\{Z_{g}\right\}\right)$. Then there exists a nonzero function $h(x, y)$ in $L_{2}\left(\Omega^{*}\right)$ such that

$$
h\left(Z_{g}(x, y)\right)=\lambda(g) \cdot h(x, y) .
$$

Let us define the function $f(\hat{y} ; x)$ as follows:

$$
f(\hat{y} ; x)=\int_{Y} h(x, y) \hat{y}(y)^{-1} d m_{Y}(y)
$$


for any $\hat{y} \in \hat{Y}$. It follows from (4.7) and (4.8) that

$$
f(\hat{y} ; x)=\hat{y}(\Delta(g, x)) \cdot \lambda(g)^{-1} \cdot f\left(\hat{y} ; T_{g} x\right) .
$$

Since $\hat{Y}$ is a complete orthonormal system of $L_{2}(Y)$, the ergodicity of $\left\{T_{g}\right\}$ assures that there exists such a character $\hat{y}_{0}$ in $\hat{Y}$ that (4.4) holds for some positive constant $c\left(\hat{y}_{0}\right)$. Thus we acquire the expression of the function $f\left(\hat{y}_{0} ; x\right)$ as in (4.5) for some $\theta(x) \in \Gamma(X)$, so that, by replacing the function $f(\hat{y} ; x)$ in (4.9) by the function $f\left(\hat{y}_{0} ; x\right)$ in $(4.5)$, we get

$$
\hat{y}_{0}(\Delta(g, x)) \lambda(g)^{-1}=\sigma\left(T_{g} x\right) \cdot \sigma(x)^{-1},
$$

where $\sigma(x)=\theta(x)^{-1} \in \Gamma(X)$, that is to say, $\hat{y}_{0}(\Delta(g, x))$ is homologous to $\lambda(g)$.

To show the converse, suppose there exists a character $\hat{y}$ in $\hat{Y}$ such that $\hat{y}(\Delta(g, x))$ is homologous to $\lambda(g)$. Then we can choose a function $\theta(x)$ in $\Gamma(X)$ for which (4.10) holds. To complete the proof, it suffices to consider the function $h(x, y)$ $=\theta(x)^{-1} \hat{y}(y)$. Indeed, it is a simple matter to show that $\lambda$ is a proper value function of $\left\{Z_{g}\right\}$ which has the corresponding proper function $h(x, y)$. Hence, the proof of the theorem is completed.

COROLlaRY 4.4. Let $\left\{T_{t}\right\}$ and $\left\{S_{t}\right\}$ be as in the hypothesis of Corollary 4.1. Furthermore, let $\left\{T_{t}\right\}$ be ergodic. The ordinary simple skew product flow $\left\{Z_{t}\right\}$ of $\left\{T_{t}\right\}$ and $\left\{S_{t}\right\}$ built up by $\alpha(t, x)$ has a proper value $\lambda$ if and only if there exists a proper value $r$ of $\left\{S_{t}\right\}$ such that $\exp i\langle r, \alpha(t, x)\rangle$ is homologous to $\exp i\langle\lambda, t\rangle$ with respect to $\left\{T_{t}\right\}$.

COROLlaRY 4.5. Let $\left\{T_{t}\right\}$ and $\left\{S_{t}\right\}$ be as in the hypothesis of Corollary 4.2. Suppose $\left\{T_{t}\right\}$ is ergodic. Then the ordinary simple skew product flow $\left\{Z_{t}\right\}$ of $\left\{T_{t}\right\}$ and $\left\{S_{t}\right\}$ built up by $\alpha(t, x)$ has a proper value $\lambda$ if and only if there exists an integer $p$ such that $\exp i \cdot p \cdot\langle a, \alpha(t, x)\rangle$ is homologous to $\exp i\langle\lambda, t\rangle$ with respect to $\left\{T_{t}\right\}$.

The following generalizes the result obtained by Anzai [1].

COROLlaRY 4.6. Let $T$ and $\left\{S_{t}\right\}$ be as in the hypothesis of Corollary 4.3, and let $T$ be ergodic. $\lambda$ is a proper value of the skew product automorphism $Z$ of $T$ and $\left\{S_{t}\right\}$ built up by $\alpha(x)$ if and only if there exists a proper value $r$ of $\left\{S_{t}\right\}$ such that $\exp i\langle r, \alpha(x)\rangle$ is homologous to $\exp i \cdot \lambda$ with respect to $T$.

THEOREM 4.3. Let $\left\{Z_{g}\right\}$ be an ergodic generalized simple skew product flow as in Theorem 4.2. A nonconstant function $h(x, y)$ in $L_{2}\left(\Omega^{*}\right)$ is a proper function of $\left\{Z_{g}\right\}$ corresponding to a proper value function $\lambda$ with $\lambda \neq 1$ if and only if there exist $a$ function $\theta(x)$ in $\Gamma(X)$ and a character $\hat{y}$ in $\hat{Y}$ such that $\hat{y}(\Delta(g, x)) \cdot \lambda(g)^{-1}$ $=\theta\left(T_{g} x\right)^{-1} \cdot \theta(x)$ and $h(x, y)=c \cdot \theta(x) \hat{y}(y)$ for some constant $c$.

Proof. Since the proof of the "if" part is clear, it is sufficient to show the "only if" part. From the hypothesis, we have the relation (4.7). Consider the function $f(\hat{y} ; x)$ defined by (4.8). Then, by virtue of (4.7) and (4.8), we obtain the equality (4.9). There exists a character $\hat{y}_{0}$ in $\hat{Y}$ such that the equality (4.4) holds for some 
positive constant $c\left(\hat{y}_{0}\right)$ because $\hat{Y}$ is a complete orthonormal system of $L_{2}(Y)$. Accordingly, we get a function $\theta(x)$ in $\Gamma(X)$ which satisfies (4.5). By using the functions $\theta(x)$ and $\hat{y}_{0}(y)$, put $\xi(x, y)=\theta(x) \cdot \hat{y}_{0}(y)$. It is not difficult to see that $\xi(x, y)$ together with the function $h(x, y)$ is also a proper function corresponding to $\lambda$. The ergodicity of $\left\{Z_{g}\right\}$ asserts that $\lambda$ is simple, namely, for some constant $c, h(x, y)$ $=c \cdot \xi(x, y)$ as was to be shown.

Corollary 4.7. Let $\left\{T_{t}: t \in R^{d}\right\}$ be an ordinary ergodic measurable flow on $\left(X, \mathscr{F}_{X}, m_{X}\right)$ with d-dimensional parameter. A function $f(x)$ in $L_{2}(X)$ is a proper function corresponding to the proper value $\lambda$ of $\left\{T_{t}\right\}$ if and only if there exists a function $\theta(x)$ in $\Gamma(X)$ such that, for some constant $c, f(x)=c \cdot \theta(x)$ and $\exp i\langle\lambda, t\rangle$ $=\theta\left(T_{t} x\right) \theta(x)^{-1}$.

This corollary generalizes the theorem due to B. O. Koopman (von Neumann $[12$, p. 625, Theorem 4]).

REMARK 4.2. Theorem 4.1, Theorem 4.2 and Theorem 4.3 established in this section are also true for skew product automorphisms. In fact, let $T$ be an automorphism of $\left(X, \mathscr{F}_{X}, m_{X}\right),\left\{S_{g}: g \in G\right\}$ a generalized canonical flow on $Y$ which is a compact Abelian group equipped with the normalized Haar measure (see Example 3.3) and $\alpha(x)$ a measurable function defined on $X$ taking values in $G$. For $T$, $\left\{S_{g}\right\}$ and $\Delta(x)=\pi(\alpha(x))$, we consider the skew product automorphism $Z$ given under the measurability condition (cf. Example 3.2) as follows: $Z(x, y)$ $=(T x, \Delta(x) \cdot y)$. Then we have the following results analogous to the theorems for flows.

(I). The skew product automorphism $Z$ is ergodic if and only if $T$ is ergodic and for any $\hat{y} \in \hat{Y}$ with $\hat{y} \neq \hat{e}, \hat{y}(\Delta(x)) \notin H(X)$.

(II). Let $T$ be ergodic. $\lambda$ is a proper value of $Z$ if and only if there exists a character $\hat{y}$ in $\hat{Y}$ such that $\hat{y}(\Delta(x))$ is homologous to $\lambda$.

(III). Suppose $Z$ is ergodic. A nonconstant function $h(x, y)$ in $L_{2}\left(\Omega^{*}\right)$ is a proper function of $Z$ corresponding to a proper value $\lambda$ with $\lambda \neq 1$ if and only if there exist a function $\theta(x)$ in $\Gamma(X)$ and a character $\hat{y}$ in $\hat{Y}$ such that $\hat{y}(\Delta(x)) \cdot \lambda^{-1}$ $=\theta(T x)^{-1} \cdot \theta(x)$ and $h(x, y)=c \cdot \theta(x) \cdot \hat{y}(y)$ for some constant $c$.

5. Pure point spectra. In this section we treat of the generalized simple skew product flow $\left\{Z_{g}\right\}$ of $\left\{T_{g}\right\}$ and $\left\{S_{g}\right\}$ built up by the Borel cycle $\Delta(g, x)$ as in the hypotheses of $\$ 4$, and we shall discuss the spectral properties of such flows.

LEMMA 5.1. Let $\left\{Z_{g}\right\}$ be ergodic. The following statements hold:

(i) the mapping $\phi: \lambda \rightarrow \hat{y}_{\lambda}$ is a homomorphism of $s\left(\left\{Z_{g}\right\}\right)$ into $\hat{Y}$,

(ii) the kernel of $\phi(\operatorname{Ker}(\phi))$ is equal to $s\left(\left\{T_{g}\right\}\right)\left({ }^{3}\right)$.

$\left(^{3}\right)$ Let $\phi_{0}$ be the homomorphism of $\{1\}$ into $s\left(\left\{T_{g}\right\}\right)$ and $\phi_{1}$ the homomorphism of $s\left(\left\{T_{g}\right\}\right)$ into $s\left(\left\{Z_{g}\right\}\right)$ given by $\phi_{1}(\lambda)=\lambda$ for any $\lambda \in s\left(\left\{T_{g}\right\}\right)$. The latter is justified by the fact that $s\left(\left\{Z_{g}\right\}\right)$ contains $s\left(\left\{T_{g}\right\}\right)$ in the natural sense. Then this lemma implies that the chain $\{1\} \stackrel{\phi_{0}}{\longrightarrow} s\left(\left\{T_{g}\right\}\right)$ $\stackrel{\phi_{1}}{\longrightarrow} s\left(\left\{Z_{g}\right\}\right) \stackrel{\oplus}{\rightarrow} \mathcal{Y}$ is an exact sequence. 
Proof of (i). In the preceding section we have shown that for any $\lambda \in s\left(\left\{Z_{g}\right\}\right)$, there exists a character $\hat{y}_{\lambda}$ in $\hat{Y}$ such that $\hat{y}_{\lambda}(\Delta(g, x))$ is homologous to $\lambda(g)$ (see Theorem 4.2). And Theorem 4.1 guarantees that for any $\lambda \in s\left(\left\{Z_{g}\right\}\right)$, there exists the unique element $\hat{y}_{\lambda}$ in $\hat{Y}$, so that the mapping $\phi$ is well defined. Suppose $\phi(\lambda)=\hat{y}_{\lambda}$ and $\phi(\mu)=\hat{y}_{\mu}$ for any $\lambda, \mu \in s\left(\left\{Z_{g}\right\}\right)$. There exist two functions $\theta(x)$ and $\sigma(x)$ in $\Gamma(X)$ such that

$$
\begin{aligned}
& \hat{y}_{\lambda}(\Delta(g, x)) \cdot \lambda(g)^{-1}=\theta\left(T_{g} x\right) \cdot \theta(x)^{-1}, \\
& \hat{y}_{\mu}(\Delta(g, x)) \cdot \mu(g)^{-1}=\sigma\left(T_{g} x\right) \cdot \sigma(x)^{-1} .
\end{aligned}
$$

Thus,

$$
\left(\hat{y}_{\lambda} \cdot \hat{y}_{\mu}\right)(\Delta(g, x))(\lambda \cdot \mu)(g)^{-1}=(\theta \cdot \sigma)\left(T_{g} x\right)(\theta \cdot \sigma)(x)^{-1} .
$$

Since $\lambda \cdot \mu \in s\left(\left\{Z_{g}\right\}\right)$, the corresponding element $\hat{y}_{\lambda \cdot \mu}$ exists in $\hat{Y}$, hereby, for some $\rho(x) \in \Gamma(X)$,

$$
\hat{y}_{\lambda \mu}(\Delta(g, x))(\lambda \mu)(g)^{-1}=\rho\left(T_{g} x\right) \rho(x)^{-1} .
$$

From (5.1) and (5.2), we get

$$
\left(\hat{y}_{\lambda \cdot \mu} / \hat{y}_{\lambda} \cdot \hat{y}_{\mu}\right)(\Delta(g, x))=(\rho / \theta \cdot \sigma)\left(T_{g} x\right)(\rho / \theta \cdot \sigma)(x)^{-1} .
$$

Again, the ergodicity of $\left\{T_{g}\right\}$ (Theorem 4.1) asserts $\hat{y}_{\lambda \mu}=\hat{y}_{\lambda} \cdot \hat{y}_{\mu}$ which implies that $\phi$ is an into homomorphism.

Proof of (ii). Let $\lambda$ belong to $\operatorname{Ker}(\phi)$. Then, for some $\theta(x) \in \Gamma(X)$,

$$
\lambda(g)^{-1}=\theta\left(T_{g} x\right) \cdot \theta(x)^{-1},
$$

and when we put $\theta^{-1}(x)=\theta(x)^{-1}, \lambda$ is a proper value function of $\left\{T_{g}\right\}$ which has the proper function $\theta^{-1}(x)$ corresponding to it, i.e. $\lambda \in s\left(\left\{T_{g}\right\}\right)$. Conversely, suppose $\lambda$ belongs to $s\left(\left\{T_{g}\right\}\right)$. This set $s\left(\left\{T_{g}\right\}\right)$ is included in the set $s\left(\left\{Z_{g}\right\}\right)$ in the natural sense. Note that there exists a function $\theta(x)$ in $\Gamma(X)$ for which (5.3) holds. In this case, the uniqueness of the correspondence between $\lambda$ and $\hat{y}_{\lambda}$ implies $\phi(\lambda)=\hat{e}$, i.e. $\lambda \in \operatorname{Ker}(\phi)$, which completes the proof.

THEOREM 5.1. Let $\left\{Z_{g}\right\}$ be ergodic. $\left\{Z_{g}\right\}$ has pure point spectrum if and only if the mapping $\phi$ is a homomorphism of $s\left(\left\{Z_{g}\right\}\right)$ onto $\hat{Y}$ and $\left\{T_{g}\right\}$ has pure point spectrum.

Proof. Suppose $\left\{Z_{g}\right\}$ has pure point spectrum. We begin by proving that the mapping $\phi$ is a homomorphism of $s\left(\left\{Z_{g}\right\}\right)$ onto $\hat{Y}$. According to Lemma 5.1, it suffices to show that $\phi$ is onto. If there exists a character $\hat{y}_{0}$ in $\hat{Y}$ such that $\hat{y}_{0}(\Delta(g, x))$ is not homologous to $\lambda(g)$ for all $\lambda \in s\left(\left\{Z_{g}\right\}\right)$, this character $\hat{y}_{0}$ is not constant and, of course, not equal to $\hat{e}$. Let $f(x, y)=\hat{y}_{0}(y)$, then $f(x, y) \in L_{2}\left(\Omega^{*}\right)$. Our assumption, combined with Theorem 4.3 , implies that the family

$$
\left\{h_{\lambda}(x, y)=c_{\lambda} \theta_{\lambda}(x) \hat{y}_{\lambda}(y): \lambda \in s\left(\left\{Z_{g}\right\}\right)\right\}
$$


turns to a complete orthogonal system of $L_{2}\left(\Omega^{*}\right)$. Clearly, we have

$$
\int_{X} \int_{Y} f(x, y) h_{\lambda}(x, y) d m_{X}(x) d m_{Y}(y)=0
$$

for all $h_{\lambda}(x, y)$ belonging to the family, from which the function $f(x, y)$ is identically equal to zero. This contradiction shows that the mapping $\phi$ is onto. Next let $\mathfrak{A}_{0}$ be the closed linear subspace of $L_{2}\left(\Omega^{*}\right)$ which is spanned by $\left\{h_{\lambda}(x, y)\right\}$ for $\hat{y}_{\lambda}=\hat{e}$. The unitary operator group $\left\{W_{g}\right\}$ on $\mathfrak{A}_{0}$ induced by $\left\{Z_{g}\right\}$ is evidently equivalent to the unitary operator group $\left\{U_{g}\right\}$ on $L_{2}(X)$ induced by $\left\{T_{g}\right\}\left({ }^{4}\right)$. Since $\left\{W_{g}\right\}$ has pure point spectrum on $L_{2}\left(\Omega^{*}\right),\left\{W_{g}\right\}$ on $\mathfrak{A}_{0}$ must have pure point spectrum. These facts show that $\left\{T_{g}\right\}$ has pure point spectrum. Conversely, to show that $\left\{Z_{g}\right\}$ has pure point spectrum, we suppose the homomorphism $\phi$ is onto and $\left\{T_{g}\right\}$ has pure point spectrum. For any character $\hat{y} \in \hat{Y}$, there exists a $\lambda$ in $s\left(\left\{Z_{g}\right\}\right)$ and a function $\theta(x)$ in $\Gamma(X)$ such that (4.10) holds. Making use of the functions $\theta(x)$ and $\hat{y}(y)$, define $h(x, y)=\sigma(x) \hat{y}(y)$, where $\sigma(x)=\theta(x)^{-1}$. Then this function $h(x, y)$ is obviously a proper function of $\left\{Z_{g}\right\}$ corresponding to $\lambda$. By $\mathfrak{A}_{1}$ denote the family

$$
\left\{\sigma(x) \hat{y}(y): \sigma(x) \in \Gamma(X), \hat{y}(y) \in \hat{Y} \text { and the pair }\left(\sigma^{-1}, \hat{y}\right)\right. \text { satisfies (4.10)\}. }
$$

Set

$$
\xi_{(\lambda, \hat{y})}(x, y)=\sigma_{\lambda}(x) \hat{y}(y), \quad \eta_{(\lambda, \mu, y)}(x, y)=\xi_{(\lambda, y)}(x, y) \cdot \xi_{(\mu, y)}(x, y)^{-1} .
$$

If $\xi_{(\lambda, y)}(x, y)$ and $\xi_{(\mu ; y)}(x, y)$ are in $\mathfrak{A}_{1}$, then we acquire, by (5.4),

$$
\eta_{(\lambda, \mu, y)}(x, y) \cdot \eta_{(\lambda, \mu, y)}\left(Z_{g}(x, y)\right)^{-1}=\left(\sigma_{\lambda} / \sigma_{\mu}\right)(x)\left(\sigma_{\lambda} / \sigma_{\mu}\right)\left(T_{g} x\right)^{-1}=(\mu / \lambda)(g) .
$$

Therefore, $\lambda \cdot \mu^{-1} \in S\left(\left\{T_{g}\right\}\right)$ and the function $\left(\sigma_{\lambda} \cdot \sigma_{\mu}^{-1}\right)(x)$ is a proper function of $\left\{T_{g}\right\}$ corresponding to $\lambda \cdot \mu^{-1}$. In this case, it is easily verified that if $\lambda \neq \mu$, then the functions $\xi_{(\lambda, y)}(x, y)$ and $\xi_{(\mu, y)}(x, y)$ are orthogonal to each other because of the ergodicity of $\left\{T_{g}\right\}$. To complete the proof of the theorem, it has to be proved that the family $\mathfrak{A}_{1}$ is complete in $L_{2}\left(\Omega^{*}\right)$. To do this, suppose that for any $f(x, y)$ $\in L_{2}\left(\Omega^{*}\right)$,

$$
\int_{X} \int_{Y} f(x, y) \sigma(x) \hat{y}(y) d m_{X}(x) d m_{Y}(y)=0
$$

holds for all $\sigma(x) \hat{y}(y) \in \mathfrak{A}_{1}$. The equality (4.10) with respect to $\sigma^{-1}$ and $\hat{y}$ and Corollary 4.7 for a generalized ergodic measurable flow show that if $\hat{y}=\hat{e}$, then $\lambda \in s\left(\left\{T_{g}\right\}\right)$, and that $\sigma(x)=\theta(x)^{-1}$ is a proper function of $\left\{T_{g}\right\}$ corresponding to $\lambda$. In such a case, we shall denote by $\sigma_{\lambda}(x)$ the proper function $\sigma(x)$. Since the flow $\left\{T_{g}\right\}$ has pure point spectrum and $s\left(\left\{T_{g}\right\}\right) \subset s\left(\left\{Z_{g}\right\}\right)$, the family

$$
\left\{\sigma_{\lambda}(x)=\theta_{\lambda}(x)^{-1}: \lambda \in s\left(\left\{T_{g}\right\}\right)\right\}
$$

$\left({ }^{4}\right)$ Here "equivalent" means "unitary equivalent", i.e. there exists a unitary operator $V$ such that for any $g \in G, V \cdot W_{g}=U_{g} \cdot V$. 
is a complete orthonormal system of $L_{2}(X)$. Hence, it follows from the facts mentioned above that (5.5) implies that $f(x, y)=0$ because $\hat{Y}$ is a complete orthonormal system of $L_{2}(Y)$. Consequently, we conclude the assertion of the theorem.

THEOREM 5.2. Let $Z$ be the ergodic skew product automorphism of $T$ and $\left\{S_{g}\right\}$ built up by $\Delta(x)$ as in Remark 4.2 of the preceding section. $Z$ has pure point spectrum if and only if the mapping $\phi: \lambda \rightarrow \hat{y}_{\lambda}$ is a homomorphism of $s(Z)$ onto $\hat{Y}$ and $T$ has pure point spectrum.

Owing to the well-known theorem of von Neumann, our results assert that an ergodic skew product dynamical system has pure point spectrum if and only if it is conjugate to the ergodic direct product dynamical system consisting of its component systems having pure point spectrum.

COROLlaRY 5.1. The generalized simple skew product flow $\left\{Z_{g}\right\}$ has pure continuous spectrum (weakly mixing property) if and only if $s\left(\left\{T_{g}\right\}\right)=\{1\}$ and $\phi\left(s\left(\left\{Z_{g}\right\}\right)\right)$ $=\{\hat{e}\}$.

COROLLARY 5.2. The skew product automorphism $Z$ has pure continuous spectrum (weakly mixing property) if and only if $s(T)=\{1\}$ and $\phi(s(Z))=\{\hat{e}\}$.

REMARK 5.1. It is to be noticed that $s\left(\left\{S_{g}\right\}\right)=\{1\}$ is not necessarily required in the Corollaries 5.1 and 5.2, and we shall mention it in the final section.

REMARK 5.2. If the skew product flow $\left\{Z_{g}\right\}$ is ergodic and has pure point spectrum, then Theorem 5.1, combined with Lemma 5.1, shows that the chain

$$
\{1\} \longrightarrow s\left(\left\{T_{g}\right\}\right) \stackrel{\phi_{1}}{\longrightarrow} s\left(\left\{Z_{g}\right\}\right) \stackrel{\phi}{\longrightarrow} \hat{Y} \longrightarrow\{1\}
$$

is an exact sequence (see footnote 3 ). However, it does not always follow that the converse holds.

6. Generalized $N$-fold skew product flows. In the sequel, we shall utilize the notations used in the preceding sections by changing the transformations or the spaces.

Let $\left(X_{k}, \mathscr{F}_{k}, m_{k}\right), 1 \leqq k \leqq N+1$ ( $N$ : a positive integer), be $\sigma$-finite measure spaces and $\left(\Omega_{N+1}^{*}, \mathscr{B}_{N+1}^{*}, m_{N+1}^{*}\right)$ the direct product measure space of the spaces. For a generalized measurable flow $\left\{T_{g}\right\}$ on $\left(X_{1}, \mathscr{F}_{1}, m_{1}\right)$, we assume that there are given the families $\left\{S_{\left(g, x_{1}, \ldots, x_{k}\right)}\right\}_{k=1}^{N}$ of transformations satisfying that, for each $k$,

(i) $S_{\left(g, x_{1}, \ldots, x_{k}\right)}$ is an automorphism of $\left(X_{k+1}, \mathscr{F}_{k+1}, m_{k+1}\right)$ for any $\left(g, x_{1}, \ldots, x_{k}\right)$ $\in G \otimes \Omega_{k}^{*}$,

(ii) $S_{\left(g_{1} g_{2}, x_{1}, \ldots, x_{k}\right)}=S_{\left(g_{2}, T_{g_{1}} x_{1}, S_{\left(g_{1}, x_{1}\right)} x_{2}, \ldots, S_{\left.\left(g_{1}, x_{1}, \ldots, x_{k-1}\right) x_{k}\right)}\right.} \times S_{\left(g_{1}, x_{1}, \ldots, x_{k}\right)}$,

(iii) both the set $\left\{\left(g, x_{1}, \ldots, x_{k+1}\right): S_{\left(g, x_{1}, \ldots, x_{k}\right)} x_{k+1} \in E\right\}$ and the set $\left\{\left(g, x_{1}, \ldots, x_{k+1}\right): S_{\left(g, x_{1}, \ldots, x_{k}\right)}^{-1} x_{k+1} \in E\right\}$ belong to $\mathscr{B} \otimes \mathscr{B}_{k+1}^{*}$, where $E$ is a $\mathscr{F}_{k+1}$-measurable set. It is easily shown that the system $\left\{Z_{g}^{(N+1)}\right\}$ of transformations which is defined by

$$
Z_{g}^{(N+1)}\left(x_{1}, \ldots, x_{N+1}\right)=\left(T_{g} x_{1}, S_{\left(g, x_{1}\right)} x_{2}, \ldots, S_{\left(g, x_{1}, \ldots, x_{N}\right)} x_{N+1}\right)
$$


is a measurable measure preserving transformation group. We call this $\left\{Z_{g}^{(N+1)}\right\}$ a generalized $N$-fold skew product flow on $\left(\Omega_{N+1}^{*}, \mathscr{B}_{N+1}^{*}, m_{N+1}^{*}\right)$. In particular, if $G=\{e\}$ and $S_{\left(g, x_{1}, \ldots, x_{k}\right)}=S_{\left(x_{1}, \ldots, x_{k}\right)}$ for each $k$, then we have a measure preserving transformation $Z_{e}^{(N+1)}$. Such a transformation $Z_{e}^{(N+1)}$ is said to be an $N$-fold skew product automorphism of the product space, and sometimes we use a symbol $Z^{(N+1)}$ instead of $Z_{e}^{(N+1)}$. From now on we suppose $\left(X_{1}, \mathscr{F}_{1}, m_{1}\right)$ is a probability measure space and, for $k \geqq 2, X_{k}$ is a compact Abelian group endowed with the normalized Haar measure $m_{k}$, on which a generalized canonical flow $\left\{S_{g}^{(k)}\right\}$ is given. And we handle the generalized $N$-fold skew product flow $\left\{Z_{g}^{(N+1)}\right\}$ given under certain measurability conditions (see (iii)) as follows:

$$
Z_{g}^{(N+1)}\left(x_{1}, \ldots, x_{N+1}\right)=\left(T_{g} x_{1}, \Delta_{1}\left(g, x_{1}\right) \cdot x_{2}, \ldots, \Delta_{N}\left(g, x_{1}, \ldots, x_{N}\right) \cdot x_{N+1}\right),
$$

where $\Delta_{1}\left(g, x_{1}\right)$ and $\Delta_{k}\left(g, x_{1}, \ldots, x_{k}\right), k \geqq 2$, are Borel cycles with respect to $\left\{T_{g}\right\}$ and $\left\{Z_{g}^{(k)}\right\}$ respectively (see Example 3.3). In the case of $G=R^{d}$, if each $X_{k}(k \geqq 2)$ is the unit interval with the usual Lebesgue measure $m_{k}$, and if the flow $\left\{S_{t}^{(k)}\right\}$ is a periodic motion on $X_{k}$ with frequency $a_{k-1}$ in $R^{d}$ defined by $S_{t}^{(k)} x_{k}=x_{k}+\left\langle a_{k-1}, t\right\rangle$ $(\bmod 1)$, then we obtain the ordinary $N$-fold skew product flow $\left\{Z_{t}^{(N+1)}\right\}$ with $d$-dimensional parameter given by

$$
\begin{aligned}
& Z_{t}^{(N+1)}\left(x_{1}, \ldots, x_{N+1}\right) \\
& \quad=\left(T_{t} x_{1}, x_{2}+\left\langle a_{1}, \alpha_{1}\left(t, x_{1}\right)\right\rangle, \ldots, x_{N+1}+\left\langle a_{N}, \alpha_{N}\left(t, x_{1}, \ldots, x_{N}\right)\right\rangle\right)(\bmod 1)
\end{aligned}
$$

where $\alpha_{1}\left(t, x_{1}\right)$ and $\alpha_{k}\left(t, x_{1}, \ldots, x_{k}\right)$ are Borel cycles with respect to $\left\{T_{t}\right\}$ and $\left\{Z_{t}^{(k)}\right\}$ respectively. Now the theorems in this section can be converted to the case of the simple skew product systems by replacing the flow $\left\{T_{g}\right\}$ by the flow $\left\{Z_{g}^{(N)}\right\}$ so that here we state only the corresponding results without their proofs.

THEOREM 6.1. A generalized $N$-fold skew product flow $\left\{Z_{g}^{(N+1)}\right\}$ given by (6.1) is ergodic if and only if the $(N-1)$-fold skew product flow $\left\{Z_{g}^{(N)}\right\}$ is ergodic and, for any nonunit character $\hat{y}_{N+1} \in \hat{X}_{N+1}, \hat{y}_{N+1}\left(\Delta_{N}\left(g, x_{1}, \ldots, x_{N}\right)\right) \notin H\left(\Omega_{N}^{*}\right)$.

Corollary 6.1. Suppose $\left\{Z_{t}^{(N+1)}\right\}$ is an ordinary $N$-fold skew product flow given by (6.2). Then $\left\{Z_{t}^{(N+1)}\right\}$ is ergodic if and only if $\left\{Z_{t}^{(N)}\right\}$ is ergodic and, for any nonzero integer $p$,

$$
\exp i p \cdot\left\langle a_{N}, \alpha_{N}\left(t, x_{1}, \ldots, x_{N}\right)\right\rangle \notin H\left(\Omega_{N}^{*}\right) .
$$

THEOREM 6.2. Let $\left\{Z_{g}^{(N+1)}\right\}$ be as in Theorem 6.1 and $\left\{Z_{g}^{(N)}\right\}$ be ergodic. $\left\{Z_{g}^{(N+1)}\right\}$ has a proper value function $\lambda$ if and only if there exists a character $\hat{y}_{N+1}$ in $\hat{X}_{N+1}$ such that $\hat{y}_{N+1}\left(\Delta_{N}\left(g, x_{1}, \ldots, x_{N}\right)\right)$ is homologous to $\lambda(g)$ with respect to $\left\{Z_{g}^{(N)}\right\}$.

Corollary 6.2. Let $\left\{Z_{t}^{(N+1)}\right\}$ be as in Corollary 6.1 and $\left\{Z_{t}^{(N)}\right\}$ be ergodic. $\lambda$ is a proper value of $\left\{Z_{t}^{(N+1)}\right\}$ if and only if there exists an integer $p$ such that

$$
\exp \text { ip } \cdot\left\langle a_{N}, \alpha_{N}\left(t, x_{1}, \ldots, x_{N}\right)\right\rangle
$$

is homologous to $\exp i\langle\lambda, t\rangle$ with respect to $\left\{Z_{t}^{(N)}\right\}$. 
THEOREM 6.3. Suppose $\left\{Z_{g}^{(N+1)}\right\}$ is as in Theorem 6.1 and is ergodic. A nonzero function $h\left(x_{1}, \ldots, x_{N+1}\right)$ in $L_{2}\left(\Omega_{N+1}^{*}\right)$ is a proper function of $\left\{Z_{g}^{(N+1)}\right\}$ corresponding to a proper value function $\lambda$ if and only if there exist a function $\theta\left(x_{1}, \ldots, x_{N}\right)$ in $\Gamma\left(\Omega_{N}^{*}\right)$ and a character $\hat{y}_{N+1}$ in $\hat{X}_{N+1}$ such that, for some constant $c$,

and

$$
h\left(x_{1}, \ldots, x_{N+1}\right)=c \cdot \theta\left(x_{1}, \ldots, x_{N}\right) \hat{y}_{N+1}(y)
$$

$$
\hat{y}_{N+1}\left(\Delta_{N}\left(g, x_{1}, \ldots, x_{N}\right)\right) \cdot \lambda(g)^{-1}=\theta\left(Z_{g}^{(N)}\left(x_{1}, \ldots, x_{N}\right)\right)^{-1} \theta\left(x_{1}, \ldots, x_{N}\right) .
$$

THEOREM 6.4. $\left\{Z_{g}^{(N+1)}\right\}$ being as in Theorem 6.3 has pure point spectrum if and only if $\left\{Z_{g}^{(N)}\right\}$ has pure point spectrum and the mapping $\phi: \lambda \rightarrow \hat{y}_{\lambda}$ is a homomorphism of $s\left(\left\{Z_{g}^{(N+1)}\right\}\right)$ onto $\hat{X}_{N+1}$.

In view of this theorem, we can conclude that an ergodic $N$-fold skew product dynamical system with pure point spectrum may be essentially regarded as an ergodic $N$-fold direct product dynamical system. Next suppose the flow $\left\{Z_{g}^{(N+1)}\right\}$ is ergodic and, for each $k, 2 \leqq k \leqq N$, the homomorphism $\phi_{k}$ of $s\left(\left\{Z_{g}^{(k)}\right\}\right)$ into $s\left(\left\{Z_{g}^{(k+1)}\right\}\right)$ is given by $\phi_{k}(\lambda)=\lambda$ for any $\lambda \in s\left(\left\{Z_{g}^{(k)}\right\}\right)$. Then one obtains an exact sequence

$$
\{1\} \longrightarrow s\left(\left\{T_{g}\right\}\right) \stackrel{\phi_{1}}{\longrightarrow} s\left(\left\{Z_{g}^{(2)}\right\}\right) \stackrel{\phi_{2}}{\longrightarrow} \cdots \stackrel{\phi_{N}}{\longrightarrow} s\left(\left\{Z_{g}^{(N+1)}\right\}\right) \stackrel{\phi}{\longrightarrow} \hat{X}_{N+1} .
$$

Further, if the ergodic flow $\left\{Z_{g}^{(N+1)}\right\}$ has pure point spectrum, then Theorem 6.4 implies that the sequence

$$
\{1\} \longrightarrow s\left(\left\{T_{g}\right\}\right) \stackrel{\phi_{1}}{\longrightarrow} s\left(\left\{Z_{g}^{(2)}\right\}\right) \stackrel{\phi_{2}}{\longrightarrow} \cdots \stackrel{\phi_{N}}{\longrightarrow} s\left(\left\{Z_{g}^{(N+1)}\right\}\right) \stackrel{\phi}{\longrightarrow} \hat{X}_{N+1} \longrightarrow\{1\}
$$

is exact (cf. Remark 5.2). If we call the number of the arrows in an exact sequence the length of the sequence, the above facts show that there exists an exact sequence with the length of an arbitrary size.

REMARK 6.1. Consider an $N$-fold skew product automorphism $Z^{(N+1)}$ given under certain measurability condition as follows:

$$
Z^{(N+1)}\left(x_{1}, \ldots, x_{N+1}\right)=\left(T x_{1}, \Delta_{1}\left(x_{1}\right) \cdot x_{2}, \ldots, \Delta_{N}\left(x_{1}, \ldots, x_{N}\right) \cdot x_{N+1}\right),
$$

where, for each $k, \alpha_{k}\left(x_{1}, \ldots, x_{k}\right)$ is a measurable function defined on $\Omega_{k}^{*}$ taking values in $G$ and $\Delta_{k}\left(x_{1}, \ldots, x_{k}\right)=\pi_{k}\left(\alpha_{k}\left(x_{1}, \ldots, x_{k}\right)\right)$. Here $\pi_{k}$, like that in Example 3.3, stands for a homomorphism of $G$ into $X_{k+1}$. The results obtained in this section have the corresponding analogs for the skew product automorphism $Z^{(N+1)}$. Take $X=X_{k}$ for each $k, 1 \leqq k \leqq N+1$, where $X$ is a compact Abelian group with the normalized Haar measure $m$ and $T$ to be an ergodic rotation on $X_{1}$. If we define

$$
Z^{(N+1)}\left(x_{1}, \ldots, x_{N+1}\right)=\left(c \cdot x_{1}, x_{1} \cdot x_{2}, \ldots, x_{1} \cdot x_{2} \ldots x_{N+1}\right)
$$

for some $c \in X_{1}$, then $Z^{(N+1)}$ becomes a model for the automorphism with quasidiscrete spectrum of the order $N+1$ (cf. Abramov [15]). 


\section{Concluding remarks.}

7.1. The entropy of ordinary skew product flows. The entropy of simple skew product automorphisms has been calculated in Adler [16], Abramov and Rohlin [17] and Newton [18]. On taking advantage of a slight modification of the method of Newton [18] using Abramov's formula [19] for a special flow, we can calculate the entropy of ordinary $N$-fold skew product flow with one-dimensional parameter. To show this, it is sufficient to consider the simple skew product flow.

Let $\left\{T_{t}: t \in R^{1}\right\}$ be a measurable flow on a normalized Lebesgue space $\left(X, \mathscr{F}_{X}, m_{X}\right)$ and $\left\{S_{t}: t \in R^{1}\right\}$ be a measurable flow on another normalized Lebesgue space $\left(Y, \mathscr{F}_{Y}, m_{Y}\right)$. For the theory of Lebesgue space and entropy of flows, refer to Rohlin [20] and Abramov [19]. Now we consider a simple skew product flow $\left\{Z_{t}\right\}$ of $\left\{T_{t}\right\}$ and $\left\{S_{t}\right\}$ built up by a Borel cycle $\alpha(t, x)$ with respect to $\left\{T_{t}\right\}$ such that $0<\alpha(1, x)<\infty$ for all $x \in X$ and $\int_{X} \alpha(1, x) d m_{X}<\infty$. We can show the following:

$$
h\left(Z_{t}\right)=h\left(T_{t}\right)+\int_{X} h\left(S_{\alpha(t, x)}\right) d m_{X}
$$

from which we see that if $\left\{T_{t}\right\}$ and $\left\{S_{t}\right\}$ have pure point spectrum, then $\left\{Z_{t}\right\}$ has zero entropy.

7.2. Isomorphism between ordinary simple skew product flows. Here we treat the case in which two spaces $X$ and $Y$ are the unit interval with the usual Lebesgue measure, and consider the periodic motion $\left\{T_{t}: t \in R^{d}\right\}$ on $X$ with frequency $a$ and the periodic motion $\left\{S_{t}: t \in R^{d}\right\}$ on $Y$ with frequency $b$. Let $\left\{Z_{t}^{(1)}\right\}$ be an ergodic ordinary simple skew product flow of $\left\{T_{t}\right\}$ and $\left\{S_{t}\right\}$ built up by a Borel cycle $\alpha(t, x)$ and $\left\{Z_{t}^{(2)}\right\}$ be another ergodic ordinary simple skew product flow of the abovestated flows built up by another Borel cycle $\beta(t, x)$. Then we have the undermentioned result which is an analogue of Anzai [1]: the flow $\left\{Z_{t}^{(1)}\right\}$ is spatially isomorphic to the flow $\left\{Z_{t}^{(2)}\right\}$ if and only if between the Borel cycles $\alpha(t, x)$ and $\beta(t, x)$ there exists the following relation:

$$
\langle b, \alpha(t, x)\rangle-\langle b, \beta(t, x+u)\rangle=\arg \theta(x)-\arg \theta\left(T_{t} x\right)(\bmod 2 \pi)
$$

or

$$
\langle b, \alpha(t, x)\rangle+\langle b, \beta(t, x+u)\rangle=\arg \theta(x)-\arg \theta\left(T_{t} x\right) \quad(\bmod 2 \pi),
$$

where $\theta(x)$ is a function of $\Gamma(X)$ and $u$ is an element of $X$. In this case, if $\Phi$ is the isomorphism, then it is given by the form

$$
\Phi(x, y)\rangle=(x+u, \arg \theta(x)+y) \quad(\bmod 2 \pi)
$$

or

$$
\Phi(x, y)\rangle=(x+u, \arg \theta(x)-y) \quad(\bmod 2 \pi) .
$$

7.3. Random ergodic theorem. Let $\left\{T_{t}: t \in R^{1}\right\}$ be a measurable flow on a finite measure space $\left(X, \mathscr{F}_{X}, m_{X}\right)$ and $\alpha(t, x)$ a Borel cycle with respect to it. Suppose there is a family $\left\{S_{\alpha(t, x)}\right\}$ of automorphisms of another finite measure space 
$\left(Y, \mathscr{F}_{Y}, m_{Y}\right)$ such that the set $\left\{(t, x, y): S_{\alpha(t, x)} y \in E\right\}$ is $\mathscr{B}^{1} \otimes \mathscr{F}_{X} \otimes \mathscr{F}_{Y}$-measurable for any $E \in \mathscr{F}_{Y}$. Then the following generalizes the Anzai result [3]:

Random Ergodic Theorem. For any function $f \in L_{p}(Y)$ with $1 \leqq p<\infty$, there exists an $m_{X}$-null set $N_{X}$ in $X$ such that for any $x \in X-N_{X}$, there exists a function $f^{*}(x, y)$ in $L_{p}(Y)$ such that

$$
\begin{aligned}
& \lim _{T \rightarrow \infty} \frac{1}{T} \int_{0}^{T} f\left(S_{\alpha(t, x)} y\right) d t=f^{*}(x, y), \\
& \lim _{r \rightarrow 0+} \frac{1}{r} \int_{0}^{r} f\left(S_{\alpha(t, x)} y\right) d t=f(y)
\end{aligned}
$$

and

$$
\lim _{T \rightarrow \infty}\left\|\frac{1}{T} \int_{0}^{T} f\left(S_{\alpha(t, x)} y\right) d t-f^{*}(x, y)\right\|_{L_{p}(Y)}=0
$$

For the further developed theory of random ergodic theorem, refer to the author [9].

7.4. Stability of Gaussian flows. The stable property coincides with the mixing property on each ergodic part of a given dynamical system.

Let $\{x(t, \omega):-\infty<t<\infty\}$ be a real measurable stationary Gaussian process on a probability space $(\Omega, \Sigma, P)$ with the mean $E\{x(t)\}=0$ for all $t$. A Gaussian flow $\left\{T_{t}\right\}$ induced by the process is given by $x\left(s, T_{t} \omega\right)=x(s+t, \omega)$ for all $t$. Let $\left\{S_{t}\right\}$ be a measurable flow on another probability space $\left(\Omega^{*}, \Sigma^{*}, P^{*}\right)$ with pure point spectrum and let us define a skew product flow $\left\{Z_{t}\right\}$ of $\left\{T_{t}\right\}$ and $\left\{S_{t}\right\}$ as follows:

$$
Z_{t}\left(\omega, \omega^{*}\right)=\left(T_{t} \omega, S_{x(t, \omega)-x(0, \omega)} \omega^{*}\right) .
$$

Then if $\left\{T_{t}\right\}$ is strongly stable, the flow $\left\{Z_{t}\right\}$ is all order stable. Although $\left\{T_{t}\right\}$ is a Kolmogorov flow and $\left\{S_{t}\right\}$ is ergodic, $\left\{Z_{t}\right\}$ is not ergodic (see Kin [4]).

Next let us consider a Brownian motion $\{x(t, \omega):-\infty<t<\infty\}$ on $(\Omega, \Sigma, P)$ and a flow $\left\{T_{t}\right\}$ of the Brownian motion which is given by $\Delta x\left(I, T_{t} \omega\right)=\Delta x(I+t, \omega)$, where $\Delta x(I, \omega)=x(b, \omega)-x(a, \omega)$ for $I=[a, b)$. If $\left\{S_{t}\right\}$ is an ergodic measurable flow on $\left(\Omega^{*}, \Sigma^{*}, P^{*}\right)$, then the skew product flow $\left\{Z_{t}\right\}$ of $\left\{T_{t}\right\}$ and $\left\{S_{t}\right\}$ given by $\left(^{*}\right)$ is strongly mixing (see Anzai [3]). Furthermore, if the ergodic flow $\left\{S_{t}\right\}$ has pure point spectrum, $\left\{Z_{t}\right\}$ is all order mixing (see Kin [4]).

These phenomena cannot be observed in the direct product dynamical systems.

\section{REFERENCES}

1. H. Anzai, Ergodic skew product transformations on the torus, Osaka Math. J. 3 (1951), 83-99. MR 12, 719.

2. Eे. Hopf, Ergodentheorie, Ergebnisse der Math., Band 5, no. 2, Springer-Verlag, Berlin, 1937, Chaps. 1-4. MR 10, 549.

3. H. Anzai, Mixing up property of Brownian motion, Osaka Math. J. 2 (1950), 51-58. MR $12,190$. 
4. E. Kin, Stable properties of Gaussian flows, Proc. Japan Acad. 46 (1970), 1116-1121.

5. S. Kakutani, Random ergodic theorems and Markoff processes with a stable distribution, Proc. Second Berkeley Sympos. Math. Statist. and Probability, 1950, Univ. of California Press, Berkeley, 1951, pp. 247-261. MR 13, 476.

6. C. Ryll-Nardzewski, On the ergodic theorems. III: The random ergodic theorem, Studia Math. 14 (1954), 298-301. MR 16, 910.

7. S. Gładysz, Ein ergodischer Satz, Studia Math. 15 (1956), 148-157. MR 18, 24.

8. - Uber den stochastischen Ergodensatz, Studia Math. 15 (1956), 158-173. MR 18, 24.

9. E. Kin, The general random ergodic theorem. I, Z. Wahrscheinlichkeitstheorie und Verw. Gebiete (to appear).

10. C. Foiaş, Automorphisms of compact abelian groups as models for measure-preserving invertible transformations, Michigan Math. J. 13 (1966), 349-352. MR 33 \#5837.

11. E. Kin, Dynamical systems of compact Abelian groups as models for non-singular flows (to appear).

12. J. von Neumann, Zur Operatorenmethode in der klassischen Mechanik, Ann. of Math. 33 (1932), 587-642.

13. H. Nomoto, On a class of metrical automorphisms on Gaussian measure space, Nagoya Math. J. 38 (1970), 21-25.

14. E. Kin, Ergodic and mixing properties of measure-preserving transformations, Proc. Japan Acad. 46 (1970), 47-50. MR 42 \#465.

15. L. M. Abramov, Metric automorphisms with quasi-discrete spectrum, Izv. Akad. Nauk SSSR Ser. Mat. 26 (1962), 513-530; English transl., Amer. Math. Soc. Transl. (2) 39 (1964), 37-56. MR 26 \#606.

16. R. L. Adler, A note on the entropy of skew product transformations, Proc. Amer. Math. Soc. 14 (1963), 665-669. MR 27 \#3779.

17. L. M. Abramov and V. A. Rohlin, The entropy of a skew product of measure-preserving transformations, Vestnik Leningrad. Univ. 17 (1962), no. 7, 5-13; English transl., Amer. Math. Soc. Transl. (2) 48 (1965), 255-265. MR 25 \#4076.

18. D. Newton, On the entropy of certain classes of skew-product transformations, Proc. Amer. Math. Soc. 21 (1969), 722-726. MR 40 \#1581.

19. L. M. Abramov, On the entropy of a flow, Dokl. Akad. Nauk SSSR 128 (1959), 873-875; English transl., Amer. Math. Soc. Transl. (2) 49 (1965), 167-170. MR 22 \#4816.

20. V. A. Rohlin, On the fundamental ideas of measure theory, Mat. Sb. 25 (67) (1949), 107-150; English transl., Amer. Math. Soc. Transl. (1) 10 (1962), 1-54. MR 11, 18; MR 13, 924.

Department of Mathematics, Tokyo Metropolitan University, Tokyo, Japan 